\title{
ON REGULATOR DESIGN FOR SPATIAL TEMPERATURE DISTRIBUTION USING FINITE-DIMENSIONAL MODELING OF HEAT EQUATIONS
}

\author{
Jun Imai ${ }^{*, 1}$ Yasuaki Ando* Masami Konishi* \\ Tatsushi Nishi * \\ * Dept. Electrical and Electronic Eng., \\ Okayama University, JAPAN
}

\begin{abstract}
A regulator problem for a heat conduction system, of which the eigenstructure is just partially known, is formulated to design a stabilizing controller that keeps a performance index less than a prescribed value. The index is made of the spatio integral of the squared deviation from reference temperature distribution. It is shown that through characterizing frequency response from input to temperature at each spatial point, a distributed parameter system with nominal model and additive uncertainty weight, both of which are real rational, is reconstructed using knowledge of the eigenstructure. A main result claims that the formulated problem is reduced to a standard mixed $H_{2} / H_{\infty}$ one for a linear finite dimensional time-invariant system. Numerical study demonstrates feasibility of the proposed design scheme. Copyright ${ }^{\circledR 2005 ~ I F A C ~}$
\end{abstract}

Keywords: Distributed-parameter systems, Uncertainty, Modeling errors

\section{INTRODUCTION}

In most applications on process temperature control, it is desired to regulate temperature on whole spatial distribution rather than ones at some measurement points. Various difficulties may occur when we formulate a problem to control such a spatially distributed variable. One of the difficulties is from infinite-dimensionality of the system described by partial differential equations, but we can cope with it by clarifying the error bound for finite dimensional approximation. We make use of finite dimensional approximation model for design because a systems (of heat conduction) is expressed as partial differential equation in principle, but in fact, its eigenstructures are

1 This work was supported by a Grant-in-Aid for Scientific Research (C)(2) from the Ministry of Education, Culture, Sorts, Science and Technology of Japan (No. 14550451). only partially known. In the studies so far, it has been established an uncertain distributed parameter system can be reconstructed using the given eigenstructures and system norm conditions(Imai et al., 2004). It is widely recognized the behavior in state distribution can be evaluated via spatio integrals; infinite-dimensional optimal regulator has been established for the case where the whole distribution can be measured. But operator Ricatti equations are needed to be solved and infinite dimensional observers are necessary to implement the regulator(Curtain and Zwart, 1995). A finite dimensional modeling technique focusing on spatial error between the system and model is proposed for flexible structures(Moheimani and Heath, 2002). Moreover they also proposed spatial control for flexible structures(Moheimani et al., 2003). The problem considered in this paper is to make the criterion function less than a 
prescribed level. Our claim is that a stabilizing controller that satisfies the condition is obtained by solving a $H_{2} / H_{\infty}$ mixed problem, which is computationally tractable. Modeling using steady state dc gain analysis enables one to keep the linear quadratic criterion bounded.

In the rest of the paper, we present system and problem formulation in chapter 2 , and how the original problem of distributed parameter systems are reduced to a standard finite dimensional Linear Matrix Inequality (LMI) in chapter 3. Chapter 4 reviews the evaluation techniques of the error bounds and chapter 5 demonstrates the feasibility of the proposed design strategy via numerical studies.

\section{SYSTEM FORMULATION}

Let us consider a control problem of temperature distribution for heat conduction system. Temperature $v(\xi, t)$ at time $t>0$ on one dimensional spatial coordinate $\xi \in[0, L]$ obeys the partial differential equation

$$
\frac{\partial v(\xi, t)}{\partial t}=\alpha^{2} \frac{\partial^{2} v(\xi, t)}{\partial \xi^{2}}-\beta v(\xi, t)+b(\xi) u(t)
$$

with boundary and initial conditions

$$
v(0, t)=v(L, t)=0, v(\xi, 0)=0
$$

and measurement output

$$
y(t)=\int_{\Omega} c(\xi) v(\xi, t) d \xi
$$

where $\alpha^{2}$ is non-negative diffusion coefficient, $\beta$ is heat transfer coefficient, and $b(\xi)$ and $c(\xi)$ are smooth non-negative functions that describe spatial heating and spatial measurement properties, respectively. Here $u(t)$ represents the input heat quantity per unite time and $y(t)$ the sensor output at time $t$.

In this paper, under the constraints that defined as in the following subsection, we aim to design a finite stabilizing compensator that generates the input by measuring the output in order to regulates errors of temperature distribution to reference.

\subsection{System model}

It is well known that a transfer function of linear time-invariant system corresponding to heat conduction and diffusion, can be written as an infinite series of first order modes:

$$
G(\xi, s)=\sum_{i=1}^{\infty} \frac{k_{i}(\xi)}{1+T_{i} s}
$$

where $k_{i}(\xi)$ is a mode coefficient and $T_{i}$ is a time constant for each $i$-th mode. Here $T_{i}>0$ and $T_{1} \geq T_{2} \geq \cdots \rightarrow 0$.
In the ideal case, all correct mode coefficients and time constants can be found, but actually only a few of relatively accurate mode coefficients and time constants are given. Then

(i) Suppose that $N$ pairs of $\left(k_{i}(\xi), T_{i}\right)$ for $i=$ $1,2, \cdots, N$ are given but all the rest (for $i=N+$ $1, \cdots)$ are unknown.

Denote the $N$-th partial sum of $G(s)$, the known part, by

$$
G_{N}(\xi, s)=\sum_{i=1}^{N} \frac{k_{i}(\xi)}{1+T_{i} s}
$$

(ii) Suppose

$$
\sum_{i=1}^{\infty}\left|k_{i}(\xi)\right| \leq \rho(\xi)
$$

for some given $\rho(\xi)>0$.

(iii) Suppose that D.C. gain

$$
G(\xi, 0)=\sum_{i=1}^{\infty} k_{i}(\xi)
$$

is given.

Consider a nominal model that consists of partial sum of $G(\xi, s)$ and compensating static deviation $G(\xi, 0)-G_{N}(\xi, 0)$ as a feedthrough term, and an additive perturbation magnitude. That is,

(a) Nominal model

$$
G_{\mathrm{nom}}(\xi, s)=G_{N}(\xi, s)+G(\xi, 0)-G_{N}(\xi, 0)
$$

(b) Additive perturbation

$$
G(\xi, s)=G_{N}(\xi, s)+\Delta(\xi, s) V(\xi) W_{\mathrm{b}}(s)
$$

where $\Delta(\xi, s) \in H^{\infty}$ with $\|\Delta(\xi, s)\|_{\infty} \leq 1$ and $\left|V(\xi) W_{\mathrm{b}}(j \omega)\right|$ denotes error bounds on frequency domain due to the finite dimensional approximation. The detail of error bounds is shown in Section 4 .

\section{CONTROLLER DESIGN}

\subsection{Problem statement}

Taking temperature distribution as controlled variable, we consider criterion function

$$
\begin{gathered}
J_{1}=\int_{0}^{\infty}\left\{\int_{\Omega} Q(\xi) \hat{v}(\xi, t)^{2} d \xi+u^{T} R u\right\} d t \\
\hat{v}(\xi, t)=v_{r}(\xi)-v(\xi, t)
\end{gathered}
$$

where $\Omega \in[0, L]$, and $v_{r}(\xi) \in \operatorname{span}\left\{k_{i}(\xi)\right\}_{i=1}^{N}$ is user-specified reference.

A compensator that makes criterion function $J_{1}$ minimum can be designed by solving a infinite dimensional Riccati equation(Curtain and Zwart, 1995). In this paper, however, we consider the problem designing a finite dimensional stabilizing compensator that makes criterion function $J_{1}$ less 
than a certain value $J$ under the constraints that the eigenstructures of the system are partially known.

\subsection{Deign of stabilizing compensator}

The next result claims that the original problem is reduced to one for a finite dimensional time invariant system, which is computationally tractable.

Theorem 1

For any uncertainty $\Delta(\xi, \cdot) \in H^{\infty}$ with $\|\Delta(\xi, \cdot)\|_{\infty} \leq 1\left(\xi^{\forall} \in \Omega\right)$, and any square integrable input $u(\cdot)$, the criterion function (10) satisfies the relation

$$
J_{1}(u) \leq I_{1}(u)
$$

where

$$
\begin{aligned}
& I_{1}=\int_{-\infty}^{\infty} \int_{\Omega}\left[2 Q ( \xi ) \left\{\left|v_{r}(\xi, j \omega)-v_{N}(\xi, j \omega)\right|^{2}+\right.\right. \\
& \left.\left.\left|V(\xi) W_{\mathrm{b}}(j \omega) u(j \omega)\right|^{2}\right\} d \xi+u(j \omega)^{T} R(j \omega) u(j \omega)\right] d \omega
\end{aligned}
$$

and $v_{N}(\xi, t)$ is partial sum of infinity series (15).

It is straightforward to show that the problem to find a controller that minimizes the criterion function $I_{1}$ can be solved by a general $H_{2}$ problem for finite dimensional linear time-invariant systems.

The aim is to design a finite dimensional stabilizing controller that regulates errors of temperature distribution to reference. Under the constraints that the eigenstructures are known partially, we can design a controller that guarantees that the original criterion function $J_{1}$ be less than a certain value $J$.

Stability should also be guaranteed, due to the error caused by truncation of unknown eigenstructures until $N$-th mode makes feedback loop unstable. The destabilization of the system is prevented by constraining a infinity norm from $w$ to $z_{\infty}$ in Fig. 2, i.e. controller $K$ needs to satisfy

$$
\left\|V\left(\xi_{o}\right) W_{\mathrm{b}}(\cdot) K\left(I+G_{N}(\cdot) K(\cdot)\right)^{-1}\right\|_{\infty}<1 .
$$

The problem that design a controller achieves two conditions: minimizes the finite dimensional criterion function and needs to be robust to the inaccuracies, can be solved by mixed $H_{2} / H_{\infty}$ problem.

\section{Proof of theorem 1 (Sketch)}

Consider a temperature distribution for controlled variable in the criterion function $(10),(11)$.

Here $v(\xi, t)$ is defined by

$$
v(\xi, t)=\sum_{i=1}^{\infty} x_{i}(t) \phi_{i}(\xi)
$$

where $\phi_{i}(\xi)$ is eigenfunctions.
The criterion function $J_{1}$ become a infinite dimensional problem, and a controller that minimize it can be designed theoretically by solving infinite dimensional Ricatti equation. However it's not always possible to obtain the eigenstructures such as eigenfunctions and eigenvalues. Therefore in this case, a controller is need to be designed under the constraints that the eigenstructures are unknown after $N$-th mode. Consequently, consider the other finite dimensional criterion function with partial sum until $N$-th mode,

$$
\begin{gathered}
J_{1}^{\prime}=\int_{0}^{\infty}\left[\int_{\Omega} Q(\xi) \hat{v}^{\prime}(\xi, t)^{2} d \xi+u^{T} R u\right] d t \\
\hat{v}^{\prime}(\xi, t)=v_{r}(\xi)-\left\{v_{N}(\xi, t)+v_{e}(\xi, t)\right\}
\end{gathered}
$$

where $v_{e}(\xi, t)$ is a error caused by truncation.

However error $v_{e}(\xi, t)$ is always unknown. Therefore we use a error bound instead of $v_{e}(\xi, t)$. Equation (16) is transformed into frequency domain using Parseval's equality, and the error bound $\bar{v}_{e}(\xi, j \omega)=\Delta(\xi, j \omega) V(\xi) W_{b}(j \omega)$ is substituted for the error $v_{e}(\xi, j \omega)$ to derive the following index $J_{1}^{\prime \prime}$ :

$$
\begin{gathered}
J_{1}^{\prime}=\frac{1}{2 \pi} \int_{-\infty}^{\infty}\left[\int_{\Omega} Q(\xi)\left|\hat{v}^{\prime}(\xi, j \omega)\right|^{2} d \xi\right. \\
\left.+u^{*}(j \omega) R u(j \omega)\right] d t \\
\hat{v}^{\prime}(\xi, j \omega)=v_{r}(\xi)-\left\{v_{N}(\xi, j \omega)+v_{e}(\xi, j \omega)\right\}
\end{gathered}
$$

$$
\begin{gathered}
J_{1}^{\prime \prime}=\frac{1}{2 \pi} \int_{-\infty}^{\infty}\left[\int_{\Omega} Q(\xi)\left|\hat{v}^{\prime \prime}(\xi, j \omega)\right|^{2} d \xi\right. \\
\left.\quad+u^{*}(j \omega) R u(j \omega)\right] d t \\
\hat{v}^{\prime \prime}(\xi, j \omega)=v_{r}(\xi)-\left\{v_{N}(\xi, j \omega)\right. \\
\left.\quad+\Delta(\xi, j \omega) V(\xi) W_{\mathrm{b}}(j \omega) u(j \omega)\right\}
\end{gathered}
$$

where $V(\xi)=\left\{\bar{\rho}^{(N)}(\xi)+\left|G(\xi, 0)-G_{N}(\xi, 0)\right|\right\} / 2$, $W_{\mathrm{b}}(j \omega)=\omega T / \sqrt{1+(\omega T)^{2}}$, and they are calculated next section. Here upper bound $J_{1}$ can be calculated by two inequalities as follows

$$
\begin{aligned}
& |A(j \omega) \pm B(j \omega)|^{2} \\
& =|A(j \omega)|^{2}+|B(j \omega)|^{2} \pm 2 \operatorname{Re}\left\{A(j \omega) B^{*}(j \omega)\right\} \\
& \leq|A(j \omega)|^{2}+|B(j \omega)|^{2}+2|A(j \omega)||B(j \omega)| \\
& \left.|| A(j \omega)|+| B(j \omega)\right|^{2} \leq\left. 2|| A(j \omega)\right|^{2}+|B(j \omega)|^{2} \mid \\
& I_{1}=\int_{-\infty}^{\infty} \int_{\Omega}\left[2 Q ( \xi ) \left\{\left|v_{r}(\xi)-v_{N}(\xi, j \omega)\right|^{2}+\right.\right. \\
& \left.\left.\left|V(\xi) W_{b}(j \omega) u(j \omega)\right|^{2}\right\} d \xi+u(j \omega)^{T} R u(j \omega)\right] d \omega
\end{aligned}
$$

An criterion function in the form of equation (24) can be solved by a general $H_{2}$ problem. Moreover $J_{1}$ and $I_{1}$ are satisfied inequality $J_{1} \leq I_{1}$, because of the inequalities $(22),(23)$. 
It is easy to see that we may have a gap between $J_{1}$ and $I_{1}$ because of the loose estimate of two inequalities (22),(23). The results below come from efforts to bridge the gap and it might improve the upper bound.

\section{Theorem 2}

Assuming $\gamma(0<\gamma \leq 1)$ which satisfying the following inequality:

$$
\begin{aligned}
\int_{\Omega}\left|V(\xi) W_{b}(j \omega) u(j \omega)\right|^{2} d \xi \leq \\
\gamma \int_{\Omega}\left|v_{r}(\xi)-v(\xi, j \omega)\right|^{2} d \xi
\end{aligned}
$$

, and for any uncertainty $\Delta(\xi, \cdot) \in H^{\infty}$ with $\|\Delta(\xi, \cdot)\|_{\infty} \leq 1\left(\xi^{\forall}\right) \in \Omega$, and any square integrable input $u(\cdot)$, the criterion function (10) satisfies the relation

$$
J_{1}(u) \leq I_{2}(u)
$$

where

$$
\begin{aligned}
& I_{1}=\frac{1}{2 \pi} \int_{-\infty}^{\infty} \int_{\Omega}\left[Q ( \xi ) \left\{\eta \cdot\left|v_{r}(\xi, j \omega)-v_{N}(\xi, j \omega)\right|^{2}\right.\right. \\
& \left.\left.+\left|V(\xi) W_{\mathrm{b}}(j \omega) u(j \omega)\right|^{2}\right\} d \xi+u(j \omega)^{*} R u(j \omega)\right] d \omega
\end{aligned}
$$

$$
\eta=\left(1+2 \gamma^{\frac{1}{2}}\right)
$$

\section{Proof of theorem 2 (Sketch)}

The finite dimensional criterion function which containing the uncertainty is represented by equation (20). Looking at spatial integration in equation (20) and using inequality (22), the following equation is obtained.

$$
\begin{aligned}
& \int_{\Omega} Q(\xi)\left|\hat{v}^{\prime \prime}(\xi, j \omega)\right|^{2} d \xi \leq \\
& \int_{\Omega} Q(\xi)\left\{\left|v(\xi)-v_{N}(\xi, j \omega)\right|^{2}+\left|V(\xi) W_{b}(j \omega) u(j \omega)\right|^{2}\right. \\
& \left.\quad+2\left|v(\xi)-v_{N}(\xi, j \omega)\right|\left|V(\xi) W_{b}(j \omega) u(j \omega)\right|\right\} d \xi
\end{aligned}
$$

Assuming $\gamma(0<\gamma \leq 1)$ which satisfying the following inequality:

$$
\begin{aligned}
& \int_{\Omega} \mid V(\xi)\left.W_{b}(j \omega) u(j \omega)\right|^{2} d \xi \leq \\
& \gamma \int_{\Omega}\left|v_{r}(\xi)-v(\xi, j \omega)\right|^{2} d \xi
\end{aligned}
$$

and apply equation (30) to Hölder's inequality, we can obtain

$$
\begin{gathered}
\int_{\Omega}\left|v(\xi)-v_{N}(\xi, j \omega)\right| \cdot\left|V(\xi) W_{b}(j \omega) u(j \omega)\right| d \xi \\
\leq\left(\int_{\Omega}\left|v(\xi)-v_{N}(\xi, j \omega)\right|^{2} d \xi\right. \\
\left.\cdot \int_{\Omega}\left|V(\xi) W_{b}(j \omega) u(j \omega)\right|^{2}\right)^{\frac{1}{2}} \\
\leq(\gamma)^{\frac{1}{2}} \int_{\Omega}\left|v(\xi)-v_{N}(\xi, j \omega)\right|^{2} .
\end{gathered}
$$

Therefore equation (29) is reduced to

$$
\begin{aligned}
& \int_{\Omega} Q(\xi)\left|\hat{v}^{\prime \prime}(\xi, j \omega)\right|^{2} d \xi \leq \\
& \int_{\Omega} Q(\xi)\left\{\eta \cdot\left|v(\xi)-v_{N}(\xi, j \omega)\right|^{2}\right. \\
& \left.+\left|V(\xi) W_{b}(j \omega) u(j \omega)\right|^{2}\right\} d \xi
\end{aligned}
$$

where $\eta=\left(1+2 \gamma^{\frac{1}{2}}\right)^{\frac{1}{2}}$. Consequently $J_{1}$ and $I_{2}$ are satisfied inequality $J_{1} \leq I_{2}$, because of the inequalities $(22),(30)$.

We can design a controller by applying the Theorem 2. Procedures for designing controller is shown as follows.

Step 1

Choose the designing parameter $\gamma$ should be determined arbitrarily.

Step 2

Design a controller $K(j \omega)$ by solving finite dimensional $H_{2} / H_{\infty}$ mixed problem.

Step 3

Find out whether the assumption, represented by inequality (25), is satisfied or not. If the assumption is not satisfied, the designing parameter $\gamma$ should be increased and go back to Step 1 .

In Step 3, we can calculate the following equation, and then we can verify whether the assumption is satisfied. A designed controller $K(j \omega)$, linear finite dimensional model $\{A, B, C, D\}$ and error bound $W_{b}(j \omega) V(\xi)$ are given, then the assumption (25) is reduced to

$$
|L(j \omega)|^{2} \leq \frac{\gamma}{M}
$$

where

$$
\begin{aligned}
& L(j \omega)=W_{b}(j \omega) K(j \omega)\{I-D K(j \omega)\}^{-1} C \\
& M=\int_{\Omega}|V(\xi)|^{2} d \xi .
\end{aligned}
$$

If inequality (32) is satisfied for all $\omega$, the assumption (25) is also satisfied. Equation (32) depends on no state variables and it is easy to calculate.

\section{CRITERION OF ERROR BOUNDS ON FREQUENCY DOMAIN}

Consider the heat conduction system (1),(2), then a transfer function can be written as an infinite series of first order lag models $G(s)=\sum_{i=1}^{\infty} k_{i} /(1+$ $\left.T_{i} s\right)$. Here $k_{i}(\xi)$ is a mode coefficient , $T_{i}>0$ is time constant for each $i$-th mode and $T_{1} \geq$ $T_{2} \geq \cdots \rightarrow 0$. A model truncated unknown part is denoted by $G_{N}(s)$. Under the constraints that $k_{i}(\xi), T_{i}$, and upper bound $\rho(\xi)$ of $\sum_{i=1}^{\infty}\left|k_{i}(\xi)\right|$ are known, the radius of possible existence bound on frequency response never less than $\bar{\rho}^{(N)}=\rho(\xi)-$ $\sum_{i=1}^{N}\left|k_{i}(\xi)\right|$ independent on frequency.

The error bound for a finite dimensional model (8) is established in literature(Imai et al., 2004) 


$$
\frac{\bar{\rho}^{(N)}(\xi)+\left|G(\xi, 0)-G_{N}(\xi, 0)\right|}{2} \cdot \frac{\omega T}{\sqrt{1+(\omega T)^{2}}}
$$

where $T \leq T_{N}$ satisfies condition $0<T_{i} \leq T(i>$ $N)$, and $\bar{\rho}^{(N)}(\xi)=\min \left\{\rho_{1}(\xi), \rho_{2}(\xi)\right\}$. Here

$$
\begin{aligned}
& \rho_{1}(\xi)=\sqrt{\int_{0}^{1} b^{2}(\zeta) d \zeta \cdot \int_{0}^{1} \bar{c}_{\xi}^{2}(\zeta) d \zeta} \\
& \rho_{2}(\xi)=\sqrt{\int_{0}^{1} \bar{b}(\zeta) b(\zeta) d \zeta \cdot \int_{0}^{1} \bar{c}_{\xi}(\zeta) c_{\xi}(\zeta) d \zeta}
\end{aligned}
$$

where $c_{\xi}(\zeta)=\delta(\zeta-\xi)$ and $\delta(\cdot)$ is Dirac's delta function. $\bar{b}(\zeta)$ is a solution to the steady state problem for the unit step input. $\overline{c_{\xi}}(\zeta)$ is for a dual problem with input spatial properties $c_{\xi}(\cdot)$.

The spatial error bound for the model with 3rd modes is depicted in Fig. 4.

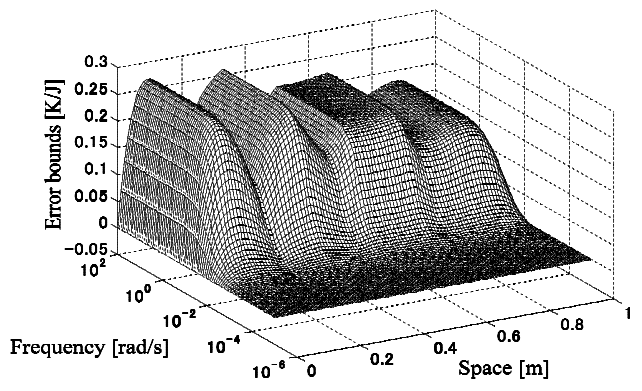

Fig. 1. Error bounds $\bar{v}_{e}(\xi, j \omega)(N=3)$

\section{NUMERICAL EXAMPLE}

\subsection{System model}

We consider again the one-dimensional heat conduction problem in equation (1) and (2). In this section we will design a stabilizing compensator regulating the spatially distributed states to a prescribed reference, and see how it works in numerical simulation.

Temperature distribution $v(\xi, t)$, at time $t>0$, on one dimensional spatial coordinate $\xi \in[0, L]$ obeys the partial differential equation

$$
\begin{gathered}
v_{t}(\xi, t)=\alpha^{2} v_{\xi \xi}(\xi, t)-\beta v(\xi, t)+b(\xi) u(t) \\
(0<\xi<L) \\
v(0, t)=v(L, t)=0 .
\end{gathered}
$$

Furthermore

$$
\begin{aligned}
y(t) & =\int_{0}^{L} c(\xi) v(\xi, t) d \xi \\
b(\xi) & = \begin{cases}\eta & \text { for } \xi \in[0, l]) \\
0 & \text { otherwise })\end{cases} \\
c(\xi) & = \begin{cases}1 / \epsilon & \text { for } \left.\left|\xi-\xi_{o}\right| \leq \epsilon / 2\right) \\
0 & \text { (otherwise) }\end{cases}
\end{aligned}
$$

where $\alpha^{2}=1.16 \times 10^{-4}\left[\mathrm{~m}^{2} / \mathrm{s}\right], L=1[\mathrm{~m}], \beta=$ $0.018[1 / \mathrm{s}], \eta=0.014\left[\mathrm{~K} / \mathrm{sV}^{2}\right], l=1 / 5[\mathrm{~m}], \epsilon=$ $1 / 500, \xi_{o}=1 / 4[\mathrm{~m}]$. The values of $\beta$ and $\eta$ are obtained by experiment using a cupper rod (Length: $1[\mathrm{~m}]$, Diameter: $2[\mathrm{~mm}]$ ). The eigenvalues $\lambda_{i}$ and eigenfunctions $\phi_{i}(\xi)$ of system (1), (2) are $\phi_{i}(\xi)=\sqrt{2} \sin (i \pi \xi), \lambda_{i}=-(i \pi)^{2}$.

\subsection{Design of controller}

Our aim is to design a controller that makes the finite criterion function (24) to minimize, and makes the feedback loop stable even taking the modeling error into account. These two criteria can be achieved solving mixed $H_{2} / H_{\infty}$ problem(Scherer, 1995).

In this paper, we proposed two finite dimensional stabilizable compensators that can be designed by solving $H_{2} / H_{\infty}$ problem. Their criterion function, respectively, give upper bounds to the original criterion function (infinite dimensional criterion function). It will be demonstrated here after by numerical example how the finite criterion functions give upper bounds to the original function is shown .

A generalized plant for controller design is shown in Fig. 2, Fig. 6. Here $V_{i}=\left(\int_{\Omega} V(\xi)^{2} d \xi\right)^{\frac{1}{2}}=$ 0.1931 in Fig. 2. Assuming that only the first three eigenvalues and eigenfunctions of system (1), (2) are known, i.e. $N=3$. In the numerical example, we regard 100 dimensional model as a plant. $H_{2} / H_{\infty}$ problem is solved by LMI Control Toolbox(Gahinet and Nemirovski, 1995). Spatial distribution of a error bound is shown in Fig. 6, and weighting functions and designing parameter using for controller design are as follows :

$$
\begin{aligned}
& Q(s)=1, \quad R(s)=0.01 \\
& W_{b}(s)=\frac{0.2078 s}{27.1606 s+1}, \gamma=0.15 .
\end{aligned}
$$

Here, assuming initial temperature distribution $v(\xi, 0)=0$, then temperature distribution is controlled to reference $v_{r}(\xi)$ that is steady state distribution that can be realized by heat source located. A result of simulation is shown in Fig 6 .

The original criterion function $J_{1}$ and the finite criterion function $I_{1}$ and $I_{2}$ can be calculated on numerical example and they are shown in Table 1. It is straightforward to see that finite criterion functions $I_{1}$ and $I_{2}$ give upper bounds, respectively, to the original criterion function $J_{1}$. Moreover criterion function $I_{2}$ can be restrained comparing to $I_{1}$, if we choose the designing parameter $\gamma$ as small as the assumption (25) is satisfied. It is difficult to solve infinite dimensional Riccati equation, and so the original criterion function was calculated by solving of order high enough finite dimensional Riccati equation. (Banks and Wang, 1989) 
Table 1 . The value of criterion function

\begin{tabular}{ccc}
\hline$J_{1}$ (Original) & $I_{1}($ Theorem 1$)$ & $I_{2}$ (Theorem 2$)$ \\
\hline $2.55 \times 10^{3}$ & $6.07 \times 10^{3}$ & $5.39 \times 10^{3}$ \\
\hline
\end{tabular}

6. CONCLUSION

We formulated a regulator problem for a heat conduction system with partially known eigenstructure to design a stabilizing controller that guarantees a performance index with spatio integral of the squared deviation in temperature distribution to be within a prescribed value. Through characterizing frequency response from input to temperature at each spatial point, a distributed parameter system with nominal model and additive uncertainty weight, both of which are real rational, was shown to be characterized just using knowledge of the eigenstructure. It was shown that a solution to a standard $H_{2} / H_{\infty}$ mixed problem for a specific linear finite dimensional time-invariant system should be the one to the originally formulated problem. Numerical study demonstrates feasibility of the proposed design scheme.

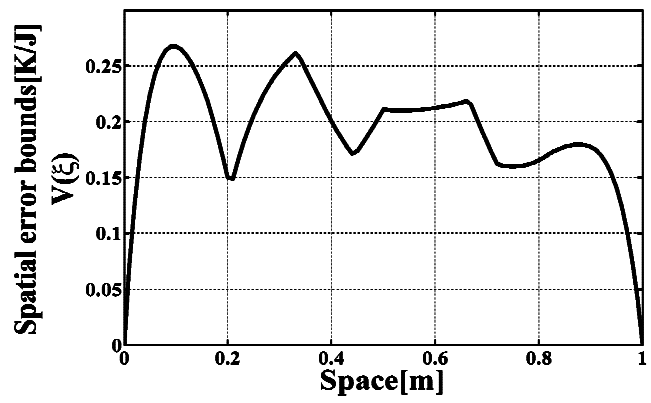

Fig. 2. Spatial error bounds $V(\xi)(N=3)$

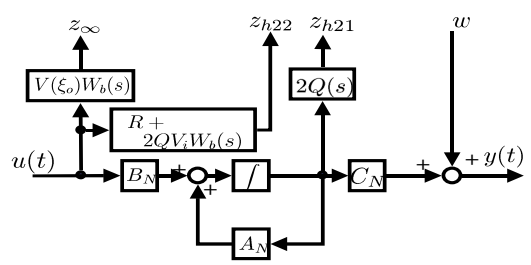

Fig. 3. Mixed $H_{2} / H_{\infty}$ solver (Theorem 1)

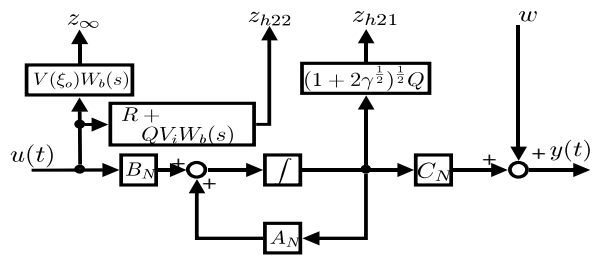

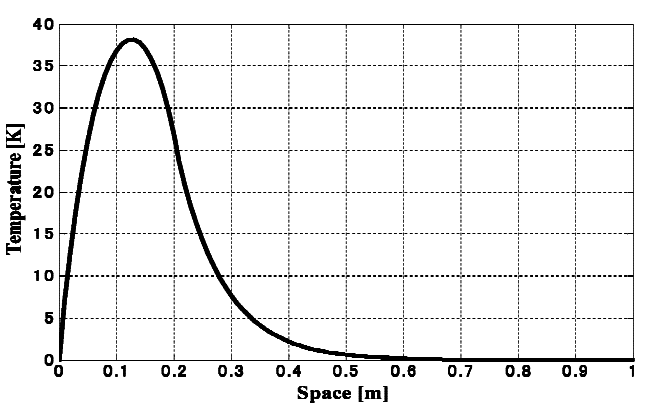

Fig. 5. Reference distribution $v_{r}(\xi)$

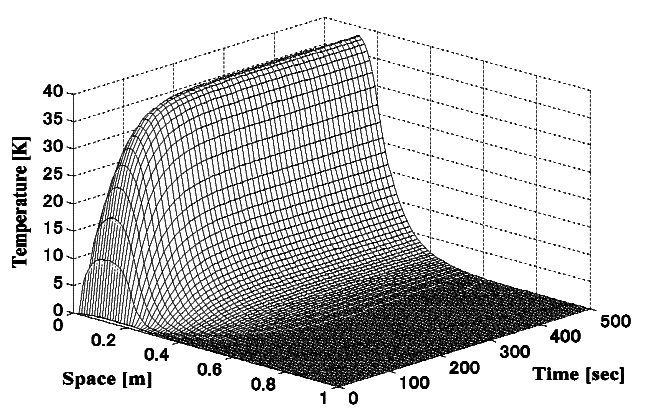

Fig. 6. Time evolution of temperature $v(\xi, t)$ REFERENCES

Banks, H. T. and C. Wang (1989). Optimal feedback control of infinite-dimensional parabolic evolution systems : approximation techniques. SIAM J. CONTROL AND OPTIMIZATION 27(5), 1182-1219.

Curtain, R. F. and H. Zwart (1995). An introduction to infinite-dimensional linear systems theory. number $21 \mathrm{In}$ : Texts in applied mathematics. Springer.

Gahinet, P. and A. Nemirovski (1995). LMI Control Toolbox User's Guide. Springer-Verlag.

Imai, J., Y. Ando and M. Konishi (2004). A finitedimensional modeling of heat conduction systems with state distribution error bounds. The $10^{\text {th }}$ IFAC/IFORS/IFIP Symposium on Large Scale Systems: Theory and Applications 2, 765-770.

Moheimani, S. O. R. and W. P. Heath (2002). Model correction for a class of spatiotemporal systems. Automatica 38, 147-155.

Moheimani, S. O. R., Dunant Halim and Andrew J. Fleming (2003). Spatial Control of Vibration. World Scientific Publishing Co. Pte. Ltd.

Scherer, C. (1995). Mixed $H_{2} / H_{\infty}$ Control. Springer-Verlag.

Fig. 4. Mixed $H_{2} / H_{\infty}$ solver (Theorem 2) 